\title{
GSM and GPS Based Real Time Vehicle Theft Tracking and Control System
}

\author{
Mr. Vinod Salunkhe ${ }^{1}$, Miss. Ghewari M.U ${ }^{2}$ \\ Student, E\&TC Department, DACOE, Karad, Maharashtra, India ${ }^{1}$ \\ Assistant Professor, E\&TC Department, DACOE, Karad, Maharashtra, India ${ }^{2}$
}

\begin{abstract}
At the present time almost of the public having an own vehicle, theft is happening on parking and sometimes driving insecurity places. The safe of vehicles is enormously essential for public vehicles. Managing location information of mobile phone devices is a very much important role in mobile computing systems. The aim of this work is to build a secured and authentic vehicle anti-theft system which will have the ability to reach the vehicle subsystems from a distal location where there is GSM network. This is the anti-theft system that not only pause your vehicle but also track the location of your lost vehicle. We have made a system which will supply users the capability to track vehicle distantly through the mobile network. Specifically, the system is supplying the owner of the vehicle to pause his moving vehicle whenever he found it riding by the new person. This is done by sending a command to the GSM modem included in the system to stop the engine.
\end{abstract}

Keywords: GSM, GPS, ARDUINO.CC Software.

\section{INTRODUCTION}

The rate of growing in car theft in this part of the world Ramani, S.Valarmathy "Vehicle Tracking and Locking has reached a dismal rate .Car thefts are growing at a System Based on GSM and GPS" described When the horrific rate all over the world. To resolve such problems, theft identified, the responsible people send SMS to the a system is cultivate using GPS and GSM technologies micro controller, then issue the control signals to stop the and an application is introduced in this project work.

engine motor.

Managing location information of mobile phone devices is This design will continuously watch a moving Vehicle and a very much important role in mobile computing systems. report the status of the Vehicle on demand

The GPS/GSM Based System is one of the most important systems, which unbroken both GSM and GPS technologies.

It is necessary due to the many of applications of both GSMand GPS systems and the large usage ofthem by millions of people throughout the world.

Vehicle owner just use his smart phone to send the off command as SMS. After switching off the engine, motor cannot restart without retransmission of SMS driven by appropriatoror by resetting the whole system.

This system is user friendly, easily accessible, easily installable, and can be used for various other purposes. After installation system will assent to track the target anytime and anywhere in any environmental conditions.

\section{LITERATURE REVIEW}

Chen, H., Chiang "REAL TIME VEHICLE CEASING AND TRACKING USING GSM AND GPS TECHNOLOGY" described To track the theft vehicle by using GPS and GSM technology. This system puts into the sleeping mode after the vehicle gets handled by the owner or authorized persons through the reset button over it.

Ch. Bhanu Prakash "Design and Implementation of a Vehicle Theft Control Unit using GSM and CAN Technology" described the present security system that will warn the owner of the vehicle by sending SMS when there has been an intrusion into the vehicle.

SMS is a good choice of the communication to replace the conventional alarm.

\section{PROPOSED SYSTEM}

In existing system drawback overcome in proposed system by using GSM technology.

In our project, we provide to track the vehicle theft by using GPS and GSM technology. when theft can be start the vehicle engine then message will send to the owner of the vehicle and also send the real time location of the stolen vehicle.

\section{BLOCK DIAGRAM}

The Proposed Block Diagram of work is shown below in fig.1- 


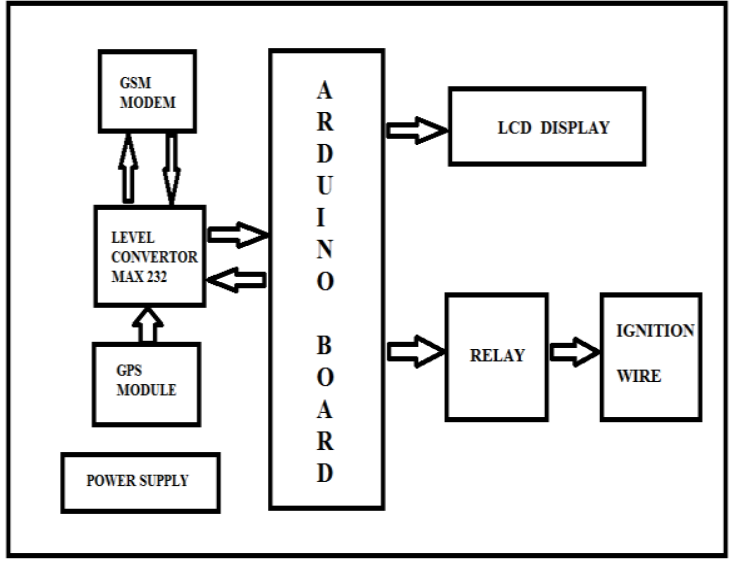

Fig.1 Block diagram of proposed system

\section{WORKING}

The block diagram of vehicle tracking and control system using GPS and GSM technology is depicted in above figure. The system processes, interfaces connect transmit the data and receives the data among the mobile unit and control station. The project consists of GPS (Global Positional System) receiver And GSM (Global System for Mobile) modem based microcontroller. The whole system is attached to the vehicle. It consists of two units, first is transmitting site that is vehicle unit and second one is monitoring unit. The compact circuitry can be built around ATmega328 microcontroller.

In this work, we proposed a system which can be used to tracking and positioning of any vehicle by using GPS (Global Positional System) and GSM (Global System for Mobile). In this system, ATmega328 microcontroller is used for interfacing various hardware peripheral. The current design is an embedded application, which will continuously monitor moving vehicle and report the status of vehicle on demand. For doing so an ATmega328 microcontroller is interfaced to GSM modem and GPS receiver.

The GPS is a satellite based navigation system of network detects vehicle. Ones the vehicle position is determined the same GPS unit can determine other information like speed, distance, time etc. GPS receiver can detects the vehicle location and provide information to responsible person by sending commands to microcontroller unit.

Our system is wireless so in wireless data transporting, GSM, SMS technology is common feature with all mobile network service provider. GSM modem is specialized type of modem which accepts a SIM card, operates on a subscriber's mobile number over a network. Vehicle owner just use his cell phone to send the "OFF" command as SMS. The LCD used for display the SMS received by owner the LCD used to display the location which is computed by the GPS receiver. LCD display device is interfaced which the microcontroller unit.
The GPS modem gives many parameter as an output which can be displayed on to the LCD. The same data is sent to mobile at the other end from where the position of vehicle is demanded. The hardware interfaces to microcontroller are LCD display, GSM modem and GPS receiver.

The ignition key is used to activate or deactivate engine of the vehicle. a) When ignition key activated, the SMS "BIKE IGNITION ON" is sent to the prescribed number. b) When the ignition key deactivated, the SMS "BIKE IGNITION OFF" is sent to the prescribed number.

When the theft is identified, the responsible people sent SMS "OFF" to the microcontroller, the issues this control signal to stop the engine motor, after that vehicle gets off and will stop. To restart the vehicle, the system should be reset. In this way, running theft with stolen vehicle can't move and have to stop at this particular instant.

\section{HARDWARE REQUIREMENTS:}

1. Arduino board

2. GPS Module

3. GSM Modem

4. Level convertor Max 232

5. Relay

6. Power supply

7. LCD Display

\section{ARDUINO BOARD :}

Arduino is an open-source platform used for building electronics projects. Arduino consists of both a physical programmable circuit board and a piece of software, or IDE (Integrated Development Environment) that runs on your computer, used to write and upload computer code to the physical board. The Arduino platform has become quite popular with people just starting out with electronics, and for good reason. Unlike most previous programmable circuit boards, the Arduino does not need a separate piece of hardware (called a programmer) in order to load new code onto the board - you can simply use a USB cable. Additionally, the Arduino IDE uses a simplified version of $\mathrm{C}++$, making it easier to learn to program. Finally, Arduino provides a standard form factor that breaks out the functions of the micro-controller into a more accessible package.

The Arduino hardware and software was designed for artists, designers, hobbyists, hackers, newbie's, and anyone interested in creating interactive objects or environments. Arduino can interact with buttons, LEDs, motors, speakers, GPS units, cameras, the internet, and even smart-phone or TV. This flexibility combined with the fact that the Arduino software is free, the hardware boards are pretty cheap, and both the software and hardware are easy to learn has led to a large community of users who have contributed code and released instructions for a huge variety of Arduino-based projects. 


\section{GPS MODULE :-}

\section{What is GPS?}

Developed by the U.S. Department of Defense for the military, the Global Positioning System (GPS) is a worldwide, satellite-based, radio navigation system that will give you the exact position of your vehicles, no matter where they are, what time it is, or what the weather is like. A total of 24 satellites orbit the Earth, monitored continuously by earth stations. The satellites transmit signals that can be detected by GPS receivers located in your vehicles and used to determine their location with great accuracy.

\section{How does GPS work?}

Each GPS satellite transmits radio signals that enable the GPS receivers in your vehicle to estimate the satellite's location, as well as the distance between it and your vehicles. The receivers then use these measurements to calculate where your vehicles are located on Earth and convert the calculations into geodetic latitude and longitude. A receiver needs signals from three GPS satellites to pinpoint your vehicle's position.

\section{GSM Modem :-}

GSM modem is a wireless modem that works with a GSM wireless network. A wireless modem behaves like a dialup modem. The main difference between them is that a dial-up modem sends and receives data through a fixed telephone line while a wireless modem sends and receives data through radio waves. Like a GSM mobile phone, a GSM modem requires a SIM card from a wireless carrier in order to operate. A GSM modem can be an external unit or a PCMCIA card (also called PC Card). An external GSM modem is connected to a PC through a serial cable, a USB cable, Bluetooth or Infrared. Like a GSM mobile phone, a GSM modem requires a SIM card from a wireless carrier in order to operate. PC's use AT commands to control a GSM modems. You can use a GSM modem just like a Hayes compatible modem. GSM modems support an extended set of AT commands. These extended AT commands are defined in the GSM standards. So signal conditioning will convert its output in to Logic Zero and Logic One. It has OPAMP as comparator.

\section{LEVEL CONVERTOR MAX 232:-}

RS-232 INTERFACE: For GSM modem which works on the RS-232 voltage levels, logic 1 varies from -3 to -15 volts and logic 0 from +3 to +15 volts. The microcontroller which works on TTL logic levels, logic 1 is +5 volts and logic 0 is 0 volts. Therefore to interface the two we use a MAX 232 driver IC manufactured by Maximum.

\section{RELAY :}

Relays are simple switches which are operated both electrically and mechanically. The switching mechanism is carried out with the help of the electromagnet. Single Pole
Single Throw (SPST) has a total of four terminals. Out of these two terminals can be connected or disconnected. The other two terminals are needed for the coil.

\section{Design of power supply}

Power supply is the major concern for every electronic device. Since the controller and other devices used are low power devices there is a need to step down the voltage and as well as rectify the output to convert the output to a constant dc. Power supply unit is the basic requirement for electronic devices.

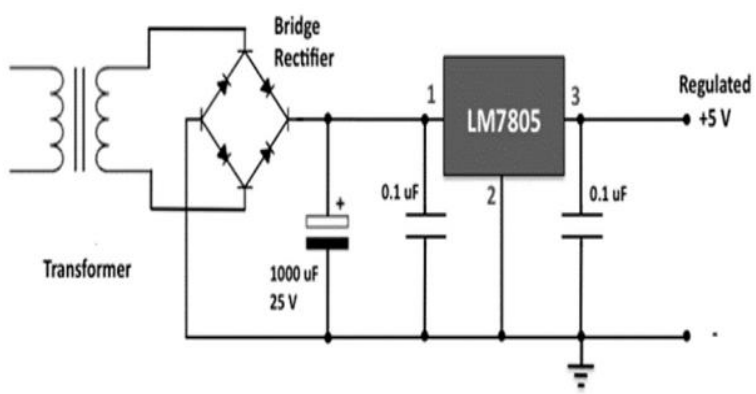

Figure 4. 1 Power supply design

\section{Voltage regulator IC 7805}

A three terminal voltage regulator is a regulator in which the output voltage is set at some predetermined value. Such regulators do not require an external feedback connection. Hence, only three terminals are required for device of such types, input (VIN) output (VO) and a ground terminal as shown in figure.

Since the regulator operates at a present output voltage the current limiting resistor is also internal to the device. The main advantages of such regulators are the simplicity of connections to the external circuit and the minimum of external components. The capacitor, at the input side is required only when the voltage regulator is located more than $5 \mathrm{~cm}$ from the power supply because the lead inductance between the supply and the regulators may cause stability problems and

High frequency oscillation. This capacitor should have very low effective resistance. Acceptable values are generally $0.33 \mu \mathrm{f}$ for ceramic disc. Although, the three terminal regulators offers only fixed output voltages, there are wide variety of voltages available, both positive and negative. The output current range from $100 \mathrm{~mA}$ to $3 \mathrm{~A}$.
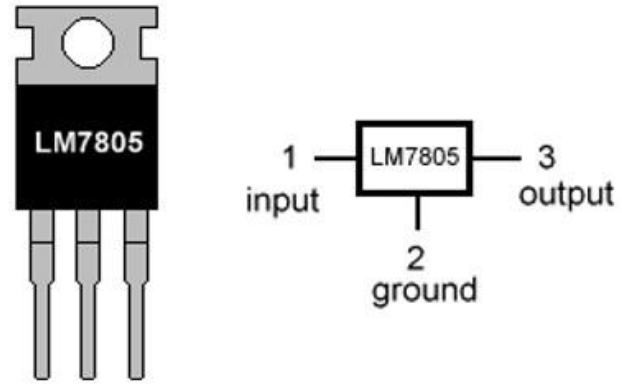

Figure 4. 2 LM 7805 
Vol. 4, Special Issue 2, January 2017

\section{Features:}

1) Output current is excess of $0.5 \mathrm{~A}$.

2) Internal thermal overload protection.

3) NO external components required.

4) Output transistor safe area protection.

5) Internal short circuit current limit.

6) Circularity all start up even if output is pulled to negative voltage.

\section{SOFTWARE REQUIREMENTS: ARDUINO SOFTWARE:-}

Arduino is a tool for making computers that can sense and control more of the physical world than your desktop computer. It's an open-source physical computing platform based on a simple microcontroller board, and a development environment for writing software for the board. Arduino can be used to develop interactive objects, taking inputs from a variety of switches or sensors, and controlling a variety of lights, motors, and other physical outputs. Arduino projects can be stand-alone, or they can be communicated with software running on your computer (e.g. Flash, Processing, Max MSP.) The boards can be assembled by hand or purchased preassembled; the opensource IDE can be downloaded for free. The Arduino programming language is an implementation of Wiring, a similar physical computing platform, which is based on the Processing multimedia programming environment.

\section{Features:-}

1) Schematic design of the open source development interface free download, and also according to the needs of their own changes

2) Download the program is simple and convenient.

3) Simply with the sensor, a wide range of electronic components connection (such as: LED light, buzzer, keypad, photo resistor, etc.), make all sorts of interesting things.

4) Using the high-speed micro-processing controller (ATMEGA328).

5) The development of language and development environment is very simple, easy to understand, very suitable for beginners to learn.

\section{ADVANTAGES:}

Real time tracking of stolen vehicle

Efficient over wide area

Avoid time consumption

Easy to access

Low Maintenance

Low Initial Costs.

\section{APPLICATION:}

1) To track the vehicle theft by using GPS and GSM technology.

2) We can locate our stolen vehicle easily using our mobile without any extra cost.

3) The device ensures vehicle security and smooth fleet management
4) We can easily install it in any vehicle such as cars, boats, and motorbikes. An SMS will inform you whether the vehicle is stationary or on the move.

5) We can also use it to keep tab on our driver.

\section{CONCLUSION}

Thus the proposed system is described that integrates new technologies offering a tracking of stolen vehicle by using GPS and GSM technology and vehicle security and smooth fleet management. The system is versatile, extendable and totally adjustable to user needs.

\section{REFERENCES}

1) Chen, H., Chiang "REAL TIME VEHICLE CEASING AND TRACKING USING GSM AND GPS TECHNOLOGY" Volume 2 Issue 1, [1]

2) R.Ramani, S.Valarmathy "Vehicle Tracking and Locking System Based on GSM and GPS" Published Online August 2013 in MECS, [2]

3) Asaad M. J, Al-Hindavi, Ibraheem Talib, “ Experimentally Evolution of GPS/GSM Based System Design", volume 2 number 2 june 2012[3]

4) Chen Peijiang, Jiang Xuehua, "Design and Implementation of Remote Monitoring System Based on GSM ", volume 42,2008[4]

5) X. Fan, W. Xu, H. Chen, and L. Liu, "CCSMOMS: A Composite Communication Scheme for Mobile Object Management System" $20^{\text {th }}$ International Conference on Advanced Information Networking and Applications, Vienna,2006,[5]

6) W.C.M Hsiao, and S. K. J. Chang, "The Optimal Location Update Strategy of Cellular Network Based Traffic Information System" In Intelligence Transportation System Conference, Toronto,2006,[6] Ch. Bhanu Prakash "Design and Implementation of a Vehicle Theft Control Unit using GSM and CAN Technology" International Journal of Innovative Research in 